\title{
Two Arguments that the Nontrivial Zeros of the Riemann Zeta Function are Irrational
}

\author{
Marek Wolf \\ Cardinal Stefan Wyszynski University \\ Faculty of Mathematics and Natural Sciences. College of Sciences \\ ul. Wóycickiego 1/3, Auditorium Maximum, (room 113) \\ PL-01-938 Warsaw, Poland \\ e-mail:m.wolf@uksw.edu.pl
}

Received: 31 October 2018; revised: 19 November 2018; accepted: 21 November 2018; published online: 07 December 2018

\begin{abstract}
We have used the first 2600 nontrivial zeros $\gamma_{l}$ of the Riemann zeta function calculated with 1000 digits accuracy and developed them into the continued fractions. We calculated the geometrical means of the denominators of these continued fractions and for all cases we get values close to the Khinchin's constant, which suggests that $\gamma_{l}$ are irrational. Next we have calculated the $n$-th square roots of the denominators $Q_{n}$ of the convergents of the continued fractions obtaining values close to the Khinchin-Lévy constant, again supporting the common opinion that $\gamma_{l}$ are irrational.
\end{abstract}

Key words: Riemann Hypothesis, zeta function, Baez-Duarte criterion

\section{INTRODUCTION}

Bernhard G.F. Riemann has shown [19] that both the sum and the product of the Euler identity which are convergent only for $\Re[s]>1$ :

$$
\begin{aligned}
\zeta(s) & :=\sum_{n=1}^{\infty} \frac{1}{n^{s}}=\prod_{p=2}^{\infty}\left(1-\frac{1}{p^{s}}\right)^{-1}, \\
s & =\sigma+i t
\end{aligned}
$$

can be analytically continued to the whole complex plane except $s=1$ by means of the following contour integral:

$$
\zeta(s)=\frac{\Gamma(-s)}{2 \pi i} \int_{\mathcal{P}} \frac{(-x)^{s}}{e^{x}-1} \frac{d x}{x}
$$

where the integration is performed along the path $\mathcal{P}$

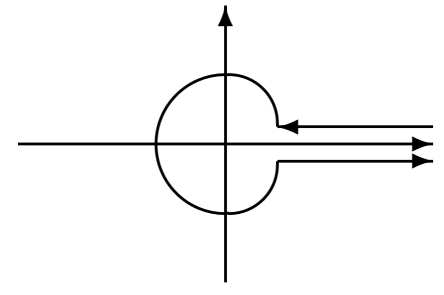

Till now dozens of integrals and series representing the $\zeta(s)$ function are known, for collection of such formulas see for example the entry Riemann Zeta Function in [24] and references cited therein.

The $\zeta(s)$ function has trivial zeros at even negative integers: $-2,-4,-6, \ldots$ and infinity of nontrivial complex ze$\operatorname{ros} \rho_{l}=\beta_{l}+i \gamma_{l}$ in the critical strip: $\beta_{l} \in(0,1), \gamma_{l} \in \mathbb{R}$. The Riemann Hypothesis (RH) asserts that $\beta_{l}=\frac{1}{2}$ for all $l$ - i.e. all zeros lie on the critical line $\Re(s)=\frac{1}{2}$. Presently it is added that these nontrivial zeros are simple: $\zeta^{\prime}\left(\rho_{l}\right) \neq 0-$ many explicit formulas of the number theory contain $\zeta^{\prime}\left(\rho_{l}\right)$ in the denominators. 
In 1914 G. H. Hardy [9] proved that infinitely many zeros of $\zeta(s)$ lie on the critical line. In 1942, A. Selberg [21] in 1942 showed that at least a (small) positive proportion of the zeros of $\zeta(s)$ lie on the critical line. The first quantitative result was obtained in 1974 by N. Levinson [12] who showed that at least one-third of the zeros lie on the critical line. In 1989, B. Conrey [2] improved this to twofifths and quite recently with collaborators [1] to over $41 \%$. It was checked computationally [8] that the $10^{13}$ first zeros of the Riemann Zeta function fulfill the condition $\beta_{l}=\frac{1}{2}$. A. Odlyzko checked that RH is not violated in different intervals around $10^{20}$ [13], $10^{21}$ [14], $10^{22}$ [17], see also [8] for the two billion zeros from the zero $10^{24}$.

There is little hope to obtain an analytical formulas for the imaginary parts $\gamma_{l}$ of the nontrivial zeros of $\zeta(s)$ but the common opinion is that they are irrational and perhaps even transcendental. In [16] Odlyzko writes: Nothing is known about the $\gamma_{n}$, but they are thought likely to be transcendental numbers, algebraically independent of any reasonable numbers that have ever been considered. The assumed existence of some hypothetical linear relations between $\gamma_{l}$ with integer coefficients appeared for the first time in the paper of A.E. Ingham [10] in connection with the Mertens conjecture. This conjecture specifies the growth of the function $M(x)$ defined by

$$
M(x)=\sum_{n \leq x} \mu(n)
$$

where $\mu(n)$ is the Möbius function

$$
\mu(n)= \begin{cases}1 & \text { for } n=1 \\ 0 & \text { when } p^{2} \mid n \\ (-1)^{r} & \text { when } n=p_{1} p_{2} \ldots p_{r}\end{cases}
$$

The Mertens conjecture asserts that

$$
|M(x)|<x^{\frac{1}{2}} .
$$

From this inequality the RH would follow. A. E. Ingham in [10] showed that the validity of the Merten's conjecture requires that the imaginary parts of the nontrivial zeros should fulfill the relations of the form:

$$
\sum_{l=1}^{N} c_{l} \gamma_{l}=0
$$

where $c_{l}$ are integers not all equal to zero. This result raised the doubts in the inequality (5) and indeed in $1985 \mathrm{~A}$. Odlyzko and H. te Riele [18] disproved the Merten's conjecture.

In [4, Th.2] P.D.T.A. Elliott assuming RH proved that the sequence $\alpha \gamma_{l}, \quad(l=1,2, \ldots)$ is uniformly distributed modulo 1 for every real nonzero $\alpha$. Further results about the distribution of $\alpha \gamma_{l}$ were later obtained in [7] and [6].
In this paper we are going to exploit two facts about the continued fractions: the existence of the Khinchin constant and Khinchin-Lévy constant, see e.g. [5, §1.8], to support the irrationality of $\gamma_{l}$. Let

$$
\begin{array}{r}
r=\left[a_{0}(r) ; a_{1}(r), a_{2}(r), a_{3}(r), \ldots\right]= \\
=a_{0}(r)+\frac{1}{a_{1}(r)+\frac{1}{a_{2}(r)+\frac{1}{a_{3}(r)+\ddots}}}
\end{array}
$$

be the continued fraction expansion of the real number $r$, where $a_{0}(r)$ is an integer and all $a_{k}(r)$ with $k \geq 1$ are positive integers. Khinchin has proved [11], see also [20], that limits of geometrical means of $a_{n}(r)$ are the same for almost all real $r$ :

$$
\begin{aligned}
& \lim _{n \rightarrow \infty}\left(a_{1}(r) \ldots a_{n}(r)\right)^{\frac{1}{n}}= \\
= & \prod_{m=1}^{\infty}\left\{1+\frac{1}{m(m+2)}\right\}^{\log _{2} m} \equiv \\
\equiv & K_{0} \approx 2.685452001 \ldots .
\end{aligned}
$$

The Lebesgue measure of (all) the exceptions is zero and include rational numbers, quadratic irrationals and some irrational numbers too, like for example the Euler constant $e=2.7182818285 \ldots$ for which the limit (8) is infinity.

The constant $K_{0}$ is called the Khinchin constant, see e.g. $[5, \S 1.8]$. If the quantities

$$
K(r ; n)=\left(a_{1}(r) a_{2}(r) \ldots a_{n}(r)\right)^{\frac{1}{n}}
$$

for a given number $r$ are close to $K_{0}$ we can regard it as an indication that $r$ is irrational.

Let the rational $P_{n} / Q_{n}$ be the $n$-th partial convergent of the continued fraction:

$$
\frac{P_{n}}{Q_{n}}=\left[a_{0} ; a_{1}, a_{2}, a_{3}, \ldots, a_{n}\right] .
$$

For almost all real numbers $r$ the denominators of the finite continued fraction approximations fulfill:

$$
\begin{aligned}
& \lim _{n \rightarrow \infty}\left(Q_{n}(r)\right)^{1 / n}=e^{\pi^{2} / 12 \ln 2} \equiv \\
\equiv & L_{0}=3.275822918721811 \ldots
\end{aligned}
$$

where $L_{0}$ is called the Khinchin-Lévy's constant [5, §1.8]. Again the set of exceptions to the above limit is of the Lebesgue measure zero and it includes rational numbers, quadratic irrational etc.

Let us remind that it is well known [22, p. 71] that the zeros of the Bessel functions $K_{\lambda}(x)$ and $J_{\lambda}(x)$ are transcendental. But $\zeta(s)$ does not belong to the class of $E$-functions considered by Siegel and his proof can not be applied to the 
zeta function. The proofs of Siegel are based on the differential equations obeyed by these functions but $\zeta(s)$ does not fulfill any differential equation. Trigonometrical functions have zeros which are transcendental, logarithms have just one integer zero. Is $\zeta(s)$ an example of a function with both integer and transcendental zeros?

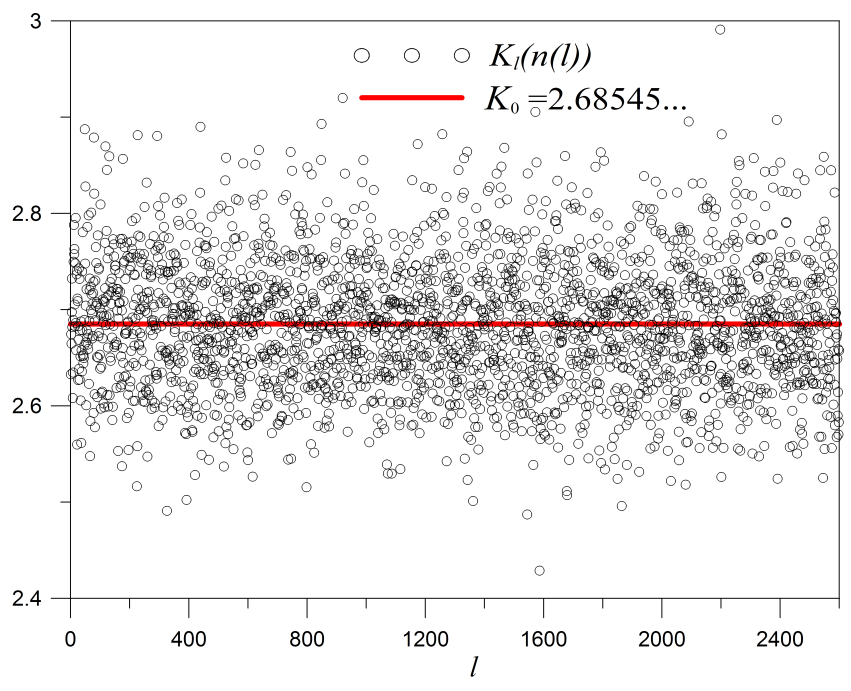

Fig. 1. The distribution of computed $K_{l}(n(l))$

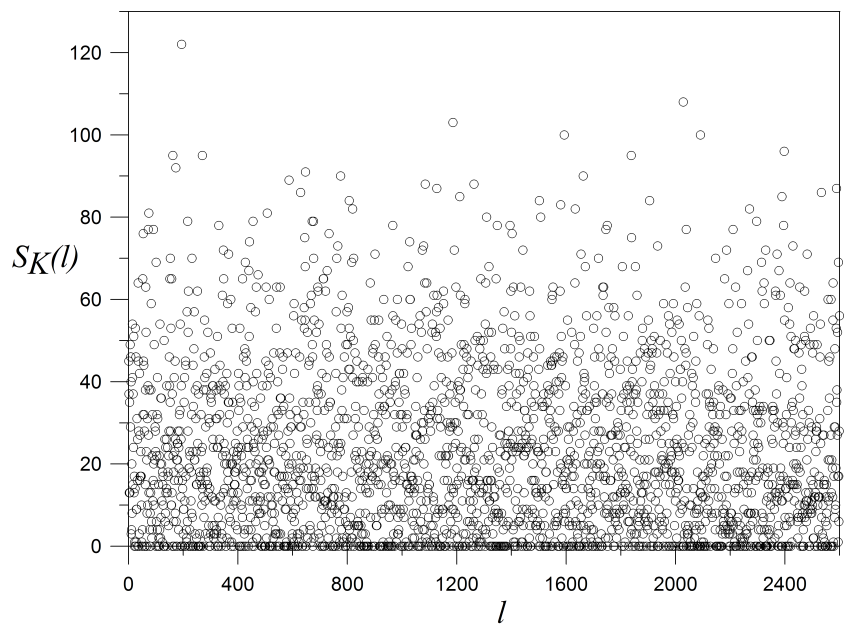

Fig. 2. The number of sign changes for each $l$, i.e. the number of such $m$ that $\left(K_{l}(m+1)-K_{0}\right)\left(K_{l}(m)-K_{0}\right)<0$ (the initial transient values of $m$ were skipped - sign changes were detected for $m=100,101, \ldots n(l))$

\section{THE COMPUTER EXPERIMENTS}

First 100 zeros $\gamma_{l}$ of $\zeta(s)$ accurate to over 1000 decimal places we have taken from [15]. Next 2500 zeros of $\zeta(s)$ with precision of 1000 digits were calculated using the built in Mathematica procedure ZetaZero[m]. We have checked using PARI/GP [23] that these zeros were accurate within at least 996 places in the sense that in the worst case $\left|\zeta\left(\rho_{l}\right)\right|<10^{-996}, l=1,2, \ldots, 2600$. PARI has built in function contfrac $(r,\{n \max \})$ which creates the row vector $\mathbf{a}(r)$ whose components are the denominators $a_{n}(r)$ of the continued fraction expansion of $r$, i.e. $\mathbf{a}=\left[a_{0}(r) ; a_{1}(r), \ldots, a_{n}(r)\right]$ means that

$$
r \approx a_{0}(r)+\frac{1}{a_{1}(r)+\frac{1}{a_{2}(r)+\frac{1}{\ddots \frac{1}{a_{n}(r)}}}}
$$

The parameter nmax limits the number of terms $a_{n \max }(r)$; if it is omitted the expansion stops with a declared precision of representation of real numbers at the last significant partial quotient: the values of the convergents $p_{k}(r) / q_{k}(r)$ approximate the value of $r$ with accuracy at least $1 / q_{k}^{2}[11$, Theorem 9, p.9]:

$$
\left|r-\frac{p_{k}}{q_{k}}\right|<\frac{1}{q_{k}^{2}}
$$

hence when $1 / q_{k}^{2}$ is smaller than the accuracy of the number $r$ the process stops. Using (simply) the trial and error approach we have determined that the PARI precision set to $\backslash p \quad 2200$ is sufficient in the sense that scripts with larger precision generated exactly the same results: the rows $\mathbf{a}\left(\gamma_{l}\right)$ obtained with accuracy 2200 digits were the same for all $l$ as those obtained for accuracy 2600 and the continued fractions accuracy set to 2100 digits gave different denominators $a_{n}\left(\gamma_{l}\right)$

With the precision set to 2200 digits we have expanded each $\gamma_{l}, l=1,2, \ldots 2600$ with 1000 accurate decimal digits value into its the continued fractions

$$
\gamma_{l} \doteq\left[a_{0}(l) ; a_{1}(l), a_{2}(l), a_{3}(l), \ldots, a_{n(l)}(l)\right] \equiv \mathbf{a}(l)
$$

without specifying the parameter nmax, thus the length of the vector $\mathbf{a}(l)$ depended on $\gamma_{l}$ and it turns out that the number of denominators was contained between 1788 and 2072. The value of the product $a_{1} a_{2} \ldots a_{n(l)}$ was typically of the order $10^{800}-10^{870}$. Next for each $l$ we have calculated the geometrical means:

$$
K_{l}(n(l))=\left(\prod_{k=1}^{n(l)} a_{k}(l)\right)^{1 / n(l)} .
$$

The results are presented in the Fig.1. Values of $K_{l}(n(l))$ are scattered around the red line representing $K_{0}$. To gain some insight into the rate of convergence of $K_{l}(n(l))$ we have plotted in the Fig. 2 the number of sign changes $S_{K}(l)$ 
of $K_{l}(m)-K_{0}$ for each $l$ when $m=100,101, \ldots n(l)$, i.e.

$$
\begin{aligned}
& S_{K}(l)=\text { number of such } m \text { that } \\
& \quad\left(K_{l}(m+1)-K_{0}\right)\left(K_{l}(m)-K_{0}\right)<0 .
\end{aligned}
$$

The largest $S_{K}(l)$ was 122 and it occurred for the zero $\gamma_{194}$ and for 381 zeros there were no sign changes at all. In the Fig. 3 we present plots of $K_{l}(m)$ as a function of $m$ for a few zeros $\gamma_{l}$.

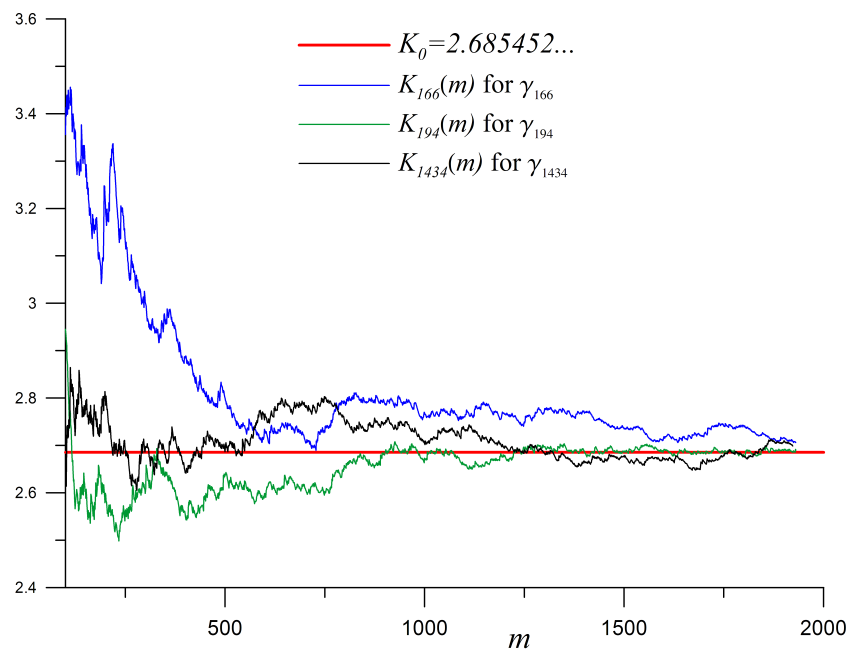

Fig. 3. For $\gamma_{166}$ there was no sign change of the difference $K_{166}(m)-K_{0}$. For $\gamma_{194}$ there were 122 sign changes of the difference $K_{194}(m)-K_{0}$-it was the largest number of sign changes among all zeros. For $\gamma_{1434}$ there were 63 sign changes of the difference $K_{1434}(m)-K_{0}$

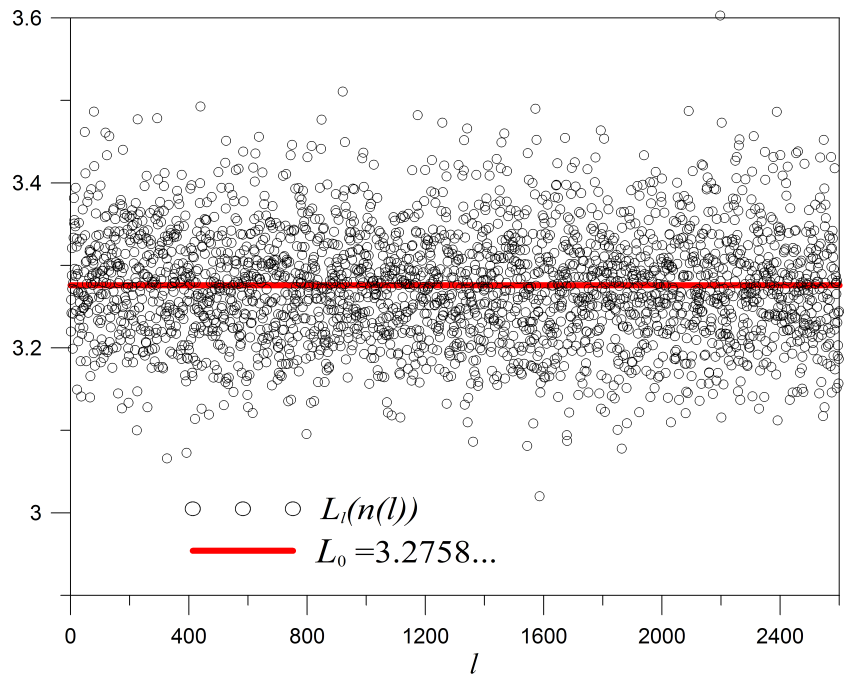

Fig. 4. The distribution of computed $L_{l}(n(l))$

Let the rational $p_{n(l)}\left(\gamma_{l}\right) / q_{n(l)}\left(\gamma_{l}\right)$ be the $n$-th partial convergent of the continued fractions (14):

$$
\frac{p_{n(l)}\left(\gamma_{l}\right)}{q_{n(l)}\left(\gamma_{l}\right)}=\mathbf{a}(l) .
$$

For each zero $\gamma_{l}$ we have calculated the partial convergents $p_{n(l)}\left(\gamma_{l}\right) / q_{n(l)}\left(\gamma_{l}\right)$. Next from these denominators $q_{n(l)}\left(\gamma_{l}\right)$ we have calculated the quantities $L_{l}(n(l))$ :

$$
L_{l}(n(l))=\left(q_{n(l)}\right)^{1 / n(l)}, \quad l=1,2, \ldots, 2600
$$

The obtained values of $L_{l}(n(l))$ are presented in the Fig.4. These values scatter around the red line representing the Khinchin-Lévy's constant $L_{0}$. As in the case of $K_{l}(m)$ Fig. 5 presents the number of sign changes of the difference $L_{l}(m)-L_{0}$ as a function of the index $m$ of the denominator of the $m$-th convergent $p_{m} / q_{m}$

$$
\begin{aligned}
& S_{L}(l)=\text { number of such } m \text { that } \\
& \quad\left(L_{l}(m+1)-L_{0}\right)\left(L_{l}(m)-L_{0}\right)<0 .
\end{aligned}
$$

The maximal number of sign changes was 136 and appeared for the zero $\gamma_{1389}$ and there were 396 zeros without sign changes.

In Fig. 6 we have plotted the "running" absolute difference between $K_{l}(m)$ and $K_{0}$ averaged over all 2600 zeros:

$$
\begin{aligned}
& A_{K}(m)=\frac{1}{2600} \sum_{l=1}^{2600}\left|K_{l}(m)-K_{0}\right| \\
& m=100,101, \ldots 1788
\end{aligned}
$$

and the similar average for the difference between $L_{l}(m)$ and $L_{0}$ :

$$
\begin{aligned}
& A_{L}(m)=\frac{1}{2600} \sum_{l=1}^{2600}\left|L_{l}(m)-L_{0}\right| \\
& m=100,101, \ldots 1788
\end{aligned}
$$

These two averages tend to zero very rapidly. Although it does not prove nothing, the fact that the curves representing $A_{K}(m)$ and $A_{L}(m)$ almost coincide is very convincing. In the inset the plot on double logarithmic scale reveals that both $A_{L}(m)$ and $A_{K}(m)$ decrease like $C_{K, L} / \sqrt{m}$ where $C_{K}=2.3868 \ldots$ and $C_{L}=2.4473 \ldots$. It would be interesting to offer the conjecture that the power of the dependencies evolves to be $m^{-(1 / 2+\epsilon)}$ with $\epsilon$ tending to zero for large $m$.

\section{CONCLUDING REMARKS}

There are generalizations of above quantities $K(n)$ given by (9). It can be shown that the following $s$-mean values of the denominators $a_{k}(r)$ of the continued fraction for a real number $r$ :

$$
M(n, s ; r)=\left(\frac{1}{n} \sum_{k=1}^{n}\left(a_{k}(r)\right)^{s}\right)^{1 / s}
$$


are divergent for $s \geq 1$ and convergent for $s<1$ for almost all real $r[5, \S 1.8]$. It can be shown that for $s<1$

$$
\begin{aligned}
& \lim _{n \rightarrow \infty} M(n, s ; r)= \\
= & \left(\sum_{k=1}^{\infty}-k^{s} \log _{2}\left(1-\frac{1}{(k+1)^{2}}\right)\right)^{1 / s} \equiv K_{s}
\end{aligned}
$$

where $M(n, s ; r)$ for almost all $r$ are the same. The quantities (22) can be computed for imaginary parts of nontrivial zeta zeros $M\left(n, s ; \gamma_{l}\right)$ and compared with values of $K_{s}$ but we leave it for further investigation.

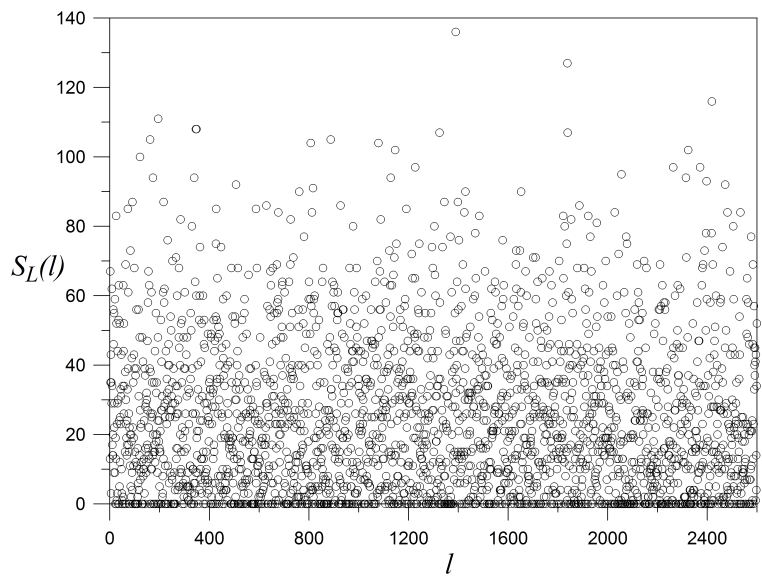

Fig. 5. The number of sign changes for each $l$. i.e. the number of such $m$ that $\left(L_{l}(m+1)-L_{0}\right)\left(L_{l}(m)-L_{0}\right)<0$ (the initial transient values of $m$ were skipped - sign changes were detected for $m=100,101, \ldots n(l))$

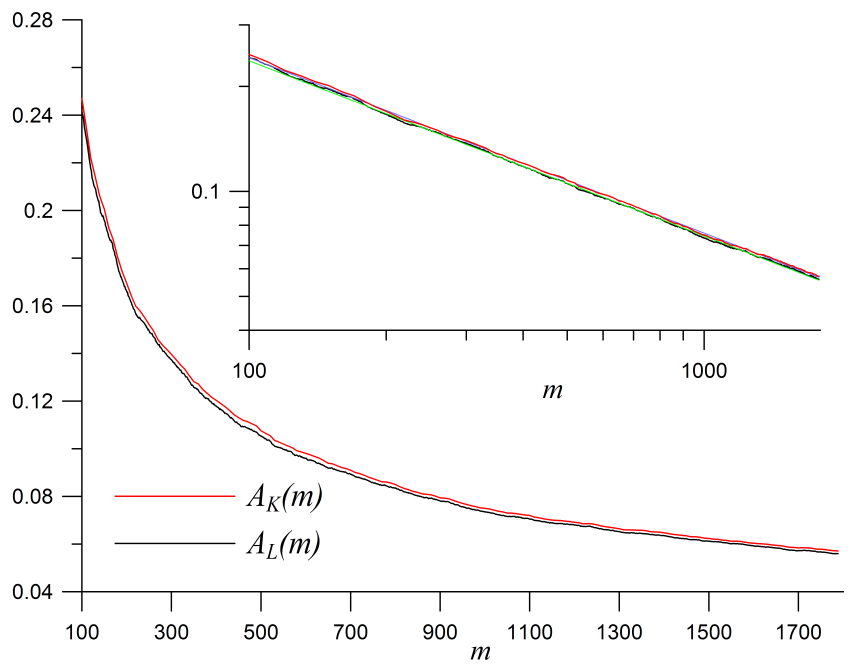

Fig. 6. The running average differences $A_{K}(m)$ (eq. (20)) and $A_{L}(m)$ (eq. (21)) plotted for $\left.m=100,101, \ldots 1788\right)$. In the inset the same curves are plotted on the double logarithmic scale (in red and black) together with fits obtained by the least square method (in blue and green). The equations of the fits are $2.3868 / \mathrm{m}^{0.5019}$ for $A_{K}(m)$ (green line) and $2.4473 / m^{0.5028}$ for $A_{L}(m)$ (blue line)
The continued fractions were used in the past in Apéry s proof of irrationality of $\zeta(3)$. In the paper [3] values of $\zeta(n)$ for all $n \geq 2$ were expressed in terms of rapidly converging continued fractions. These results were analytical, but in case of the nontrivial zeros of the $\zeta(s)$ function we are left only with the computer experiments. The results reported in this paper suggest that they are irrational.

\section{Acknowledgement}

The author wishes to thank the referees for their suggestions to improve the presentation.

\section{References}

[1] B. Conrey, H.M. Bui, M.P. Young, More than $41 \%$ of the zeros of the zeta function are on the critical line, Acta Arithmetica 150, 35-64 (2011).

[2] J.B. Conrey, More than two-fifths of the zeros of the Riemann zeta function are on the critical line, J. Reine Angew. Math. 399, 1-26 (1989).

[3] D. Cvijović, J. Klinowski, Continued-Fraction Expansions for the Riemann Zeta Function and Polylogarithms, Proceedings of the American Mathematical Society 125(9), 25432550 (1997).

[4] P. Elliott, The Riemann Zeta function and coin tossing, Journal für die reine und angewandte Mathematik, 254, 100-109 (1972).

[5] S.R. Finch, Mathematical Constants, Cambridge University Press, 2003.

[6] K. Ford, K. Soundararajan, A. Zaharescu, On the distribution of imaginary parts of zeros of the riemann zeta function, ii, Mathematische Annalen 343(3), 487-505 (2009).

[7] K. Ford and A. Zaharescu, On the distribution of imaginary parts of zeros of the riemann zeta function, Journal für die reine und angewandte Mathematik 2005, 03 (2005).

[8] X. Gourdon, The $10^{13}$ first zeros of the Riemann Zeta Function, and zeros computation at very large height, Oct. 24, 2004. http://numbers.computation.free.fr/Constants/ Miscellaneous/zetazeros1e13-1e24.pdf.

[9] G. H. Hardy, Sur les zéros de la fonction $\zeta(s)$ de Riemann, C. R. Acad. Sci. Paris 158, 1012-1014 (1914).

[10] A.E. Ingham, On two conjectures in the theory of numbers, Amer. J. Math. 64, 313-319 (1942).

[11] A.Y. Khinchin, Continued Fractions, Dover Publications, New York, 1997.

[12] N. Levinson, More than one third of zeros of Riemann's zetafunction are on $\sigma=1 / 2$, Advances in Math. 13, 383-436 (1974).

[13] A.M. Odlyzko, The $10^{20}$-th zero of the Riemann zeta function and 175 million of its neighbors, 1992 revision of 1989 manuscript.

[14] A.M. Odlyzko, The $10^{21}$-st zero of the Riemann zeta function, Nov. 1998 note for the informal proceedings of the Sept. 1998 conference on the zeta function at the Edwin Schroedinger Institute in Vienna.

[15] A.M. Odlyzko, Tables of zeros of the Riemann zeta function, http://www.dtc.umn.edu/ odlyzko/zeta_tables/index.html. 
[16] A.M. Odlyzko, Primes, quantum chaos, and computers, Number Theory, National Research Council, pages 35-46, 1990.

[17] A.M. Odlyzko, The $10^{22}$-nd zero of the Riemann zeta function, In M. van Frankenhuysen, M.L. Lapidus, editors, Dynamical, Spectral, and Arithmetic Zeta Functions, number 290 in Amer. Math. Soc., Contemporary Math. series, pages 139-144, 2001.

[18] A.M. Odlyzko, H.J.J. te Riele, Disproof of the Mertens Conjecture, J. Reine Angew. Math. 357, 138-160 (1985).

[19] B. Riemann, Ueber die anzahl der primzahlen unter einer gegebenen grösse, Monatsberichte der Berliner Akademie, pages 671-680, November 1859, english translation available at http://www.maths.tcd.ie/pub/Hist Math/People/Riemann.

[20] C. Ryll-Nardzewski, On the ergodic theorems II (Ergodic theory of continued fractions), Studia Mathematica 12, 7479 (1951).

[21] A. Selberg, On the zeros of Riemann's zeta-function, Skr. Norske Vid. Akad. Oslo I. 10 59, (1942).

[22] C.L. Siegel, Transcendental Numbers, Princeton University Press, 1949.

[23] The PARI Group, Bordeaux, PARI/GP, version 2.3 . 2, 2008, available from http://pari.math.u-bordeaux.fr/.

[24] E.W. Weisstein, CRC Concise Encyclopedia of Mathematics, Chapman \& Hall/CRC, 2009.

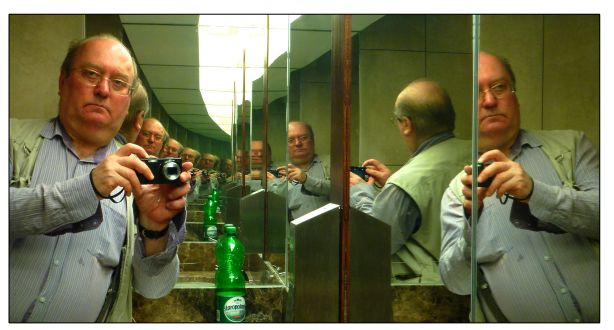

Marek Wolf, obtained his PhD degree in Physics(1982) and habilitation (1993) at Wroclaw University, where he was employed till 2011. Since 1984 he has been doing computer experiments in physics and mathematics. In 1991 and 1993 he was a research fellow at the Center for Polymer Studies at Boston University. Now he is a member of the Faculty of Mathematics and Natural Sciences, College of Sciences at the Cardinal Stefan Wyszynski University in Warsaw. His hobby is photography: he owns a few camera bodies, 15 lenses and 6 flashes. 